\title{
Evaluation of Flow Dependent External Costs in Freight Logistics Networks
}

\author{
Daniela Ambrosino ${ }^{a}$, Anna Sciomachen ${ }^{a}$, Chiara Surace $^{b}$ \\ a: Department of Economics and Business Studies, University of Genoa, Italy \\ Email: ambrosin@economia.unige.it,sciomach@economia.unige.it \\ b: Department of Mathematics, University of Genoa, Italy \\ E-mail: chiara.surace@ hotmail.it
}

\begin{abstract}
In this paper we face a distribution problem arising in a freight logistics context. More precisely, we are involved with the containerized flow originating from maritime terminals and going to inland destinations using the road transportation network. The goal is the minimization of the total shipping costs, given by the travelled distance, vehicles and external cost components. In particular, accidental and polluting costs are considered as external negative costs impacting on the collectivity. Note that the proposed externality costs depend on the amount of flow through the selected arcs in the route and their capacity; therefore, a novel stepwise function is proposed and analyzed. A deep analysis is performed for evaluating the impact of environmental externalities on the choice of the route in terms of costs and distances depending on the density of the traffic. Results of an extensive computational experimentation performed with randomly generated instances of different size and capacity values of the arcs are reported. A real case instance related to the logistics network connecting the main ports of the Liguria County (Italy) to the main inland destinations is finally proposed and analyzed.
\end{abstract}

Keywords. Logistics networks; Containerized flow; Sustainability; $o-d$ paths; Min cost flow problem; External costs.

\section{Introduction.}

Recently, sustainability, in particular in transport and logistics, is one of the most discussed topics in the economic, social and environmental fields. Talking about sustainability means discussing compatibility between the development of economic activities and the preservation of the environment. In fact, transport and logistics generate not only direct private costs but also generalized ones due to the potential negative externalities. The main types of externalities that generate costs, according to the data reported in the European Union's manual [23] are: accidents, air pollution, climate change, congestion and noise.

Without policy intervention, these costs, that is the external ones, are not taken into account by users when making transport decisions. The assessment of the external costs generated by freight transport is of fundamental importance as support for the decision-making and evaluation process of both transport policies and investment projects for logistics and infrastructures. More specifically, the issue of introducing remedial measures that consider external costs through their internalization into the overall financial price due to transport and logistics services has become a crucial aspect of transport policies and research [22, 25]. A thorough review of road transport externalities and economic instruments to internalize them is provided in Santos et al. (2010) [24].

Indeed, many research works recently proposed in the literature have the aim of quantifying the costs needed to achieve a more sustainable worldwide logistics system than the current one. However, cost estimations varied over the time due to the difficulties in clearly evaluating external effects and 
their impact. In particular, external costs, including environmental components, enhance the divergence between the perceived private costs of a specific economic activity and the real overall costs.

Sustainable development is a key point in the European Union's environmental actions and policies [11]; for this reason, external costs have recently been deeply studied under many EU projects, aiming, for instance, at assuring their internalization. In particular, the Handbook on estimation of external costs for the transport sector by Maibach et al. (2008) [18] constituted one of the first attempts to systematize external costs by evaluating and comparing them over large regions.

Together with the actions proposed by the European Union, many studies tried to evaluate the discrepancy between the private perceived costs of a specific transport solution and its actual cost for the society [15]. Sustainability is therefore a topic of great importance in many research fields, including Operations Research, Transport Engineering and Transport Economy [8, 16, 20]. Today's challenge is to see how researchers in these areas can contribute to the development of models and methods that lead to decision-making processes based on sustainability goals in transport and logistics. In this direction, a number of papers on urban distribution have been recently published with the aim of providing advances for the development of a sustainable city logistics (see [9], [6] and [27] among others). In particular, some authors focus on the impact of environmental factors on routing and distribution problems in urban area $[5,7,14]$. Sustainability applied to supply chain management is another emerging research field in the last decade [4, 13, 26].

Considering logistics networks, recent studies underline that the freight transport will have a positive trend in the next years, increasing until 2050 throughout Europe by $80 \%$ [3]. In this scenario, the importance of ports as international logistic nodes will increase too; therefore, higher freight flows among ports and hinterlands must be even more efficiently managed. Note that connections between a port and its hinterland are not just important in terms of efficiency but also for the overall costs due to different transport solutions, accounting not only for direct private costs but also for external ones $[3,10,12]$. In this context, it is strongly required for the road transportation network to be made more sustainable and, at the same time, for transport policies to be able to shift part of the volumes destined to road transport to other modalities [3, 17]. In [1] policies for incentivizing modal split in favor of the rail transportation are proposed; such policies are based on the internalization of the externalities. Recent literature focused its discussion on the effective flow distribution in a determined hinterland (e.g. [11, 19]) and on the benefits of the internalization of transport costs [2].

However, in many countries, in particular in Italy, road transport is still the most convenient shipping modality. Therefore, there is a real need of making evidence of the social costs derived from such a high increasing volumes of goods travelling every day on the main highway connections, merging them with the private and commercial vehicles.

In this paper we focus on the containerized flow coming from maritime terminals and shipped toward inland destinations via road modality. More precisely, we consider a multi origins - multi destinations min cost flow problem, where the objective function has some flow dependent cost components, representing the externalities which increase with the density of traffic. In particular, we consider pollution and accidental costs as external cost components and see how these cost components can affect the resolution of the multi origins - multi destinations min cost flow problem under consideration. To the best of the authors' knowledge this is the first attempt to explicitly evaluate the choice of the shipping companies to deliver containerized goods via road in terms externalities, such as pollution and accidents. 
The organization of the paper is as follows. Section 2 gives a detailed description of the present problem, together with a definition of its related underlying network model and the formulation of the resulting optimization problem. Furthermore, a whole subsection is devoted to the description of the external cost components considered in this work. Section 3 reports our computational experimentation based on both opportunely randomly generated instances and a real case concerning the logistics network connecting the main ports of the Liguria county to the main inland destinations. Finally, some conclusions and outlines for future works are given.

\section{Problem definition and formulation.}

\subsection{The freight logistics network model.}

The real system we are dealing with is a freight logistics network consisting of road connections. The goal is to optimize the containerized flow of vehicles, i.e. trucks, travelling on road transportation networks. More precisely, we have to determine how to route trucks from ports to inland destinations having to minimize the overall shipping costs while satisfying the demand at the destinations nodes, the volume of containerized flow to deliver from ports and the road capacity limits.

The freight logistics network under study is modelled by a digraph $G=(N, A)$, where $N$ is the set of $n$ nodes partitioned into three subsets, namely set $P$ of ports, set $D$ of destinations and set $T$ of transition nodes. Therefore, $N=P \cup D \cup T$, such that $P \cap D \cap T=\varnothing$.

$A$ is the set of $m$ arcs representing direct connections between nodes. Since road traffic is evaluated along one direction, let us focus, without loss of generality, only on the containerized import flow going from ports to the hinterland. In this way, given a node $p \in P$ and a node $t \in T$, we can have $\operatorname{arc}(p, t) \in A$, while $\operatorname{arc}(t, p) \notin A$. However, note that node $d, \forall d \in D$, can have both outgoing and ingoing arcs, since a destination node can be a transit node for some flow to be forwarded to a destination farther away from the origin port than the location at node $d$.

All origin nodes $p \in P$ have a value $g_{p}$ associated with them representing the amount of units of containerized flow available at port $p$; analogously, all destination nodes $d \in D$ have a value $-g_{d}$ associated with them representing the required amount of units of goods to be shipped at destination $d$. We assume that the total amount of containerized flow $b$ circulating through the network is such that $b=\sum_{p=1}^{|P|} g_{p}=\sum_{d=1}^{|D|}-g_{d}$.

Furthermore, each arc $(i, j) \in A$ has a capacity $q_{i j}$ representing the maximum amount of containerized flow $x_{i j}$ allowed to transit along arc $(i, j) \in A$. The values to assign to the $m$-dimensional vector $\underline{x}$ are our decision variables.

Finally, a cost $c_{i j}$ is associated with each arc $(i, j) \in A$, expressing the corresponding travelling cost; more precisely, $c_{i j}=c \cdot l_{i j}$, where the value $l_{i j}$ associated with each arc $(i, j) \in A$ represents the distance between nodes $i$ and $j$ and $c$ is a given constant value, which depends on the type of vehicle used for the shipping service. Thus, the total cost for shipping the flow from ports to destinations can be expressed as:

$$
C=f(\underline{x})=c \cdot \sum_{(i, j) \in A} l_{i j} \cdot x_{i j}
$$




\subsection{Definition of the external cost components.}

In the present study, we analyze the external costs related to pollutants' emissions and accidents in the case of road transport of goods. In order to perform our analysis we started from the manual published by the European Union [18], in which external cost data of all the countries of the Union are reported. Furthermore, we refer to the research article by Prez-Martínez and Vassallo-Magro (2013) [21], where a deep study related to the external costs in the Spanish transportation network is proposed. More precisely, the data used in [21] have been considered as our starting point and successively properly adapted to the Italian road transportation network. The values considered are on a yearly basis and refer to the last update version of the EU manual [23].

As already said, in our study we consider as transport modality the road one; more precisely, we refer to diesel powered trucks. Based on [21], the external costs related to pollution and accidents are computed as follows.

First, let us define the inter-urban energy consumption $E$, expressed in tera-joule, as:

$$
E=f \cdot N C V \cdot I
$$

where $f$ is the fuel consumption, expressed in grams of equivalent oil per vehicle per kilometer, $N C V=0,454 \mathrm{MJ} /$ goe (mega-joules per gram of equivalent oil) represents the net calories of the considered fuel, that is diesel, and $I$ is the traffic intensity, expressed in millions of vehicles per kilometer. From EUROSTAT [29] we derived that $I=112.637$ Mveh-km.

Following a document produced by the Italian Petroleum Union [32], it has been possible to know that the yearly diesel consumption is $f=23249 \cdot 10^{3} t=23.24910^{3} \cdot 1,017$ toe $=23.644,23310^{3}$ tons of equivalent oil; therefore, in the present work we assign to $E$ the value $E=10734.482 \cdot 10^{3}$ (TJ). Then, let us define the $\mathrm{CO}_{2}$ emission, expressed in tons of equivalent $\mathrm{CO}_{2}$, as:

$$
Z=E \cdot C E F
$$

where $C E F$ is the coal related factor of the considered fuel, expressed in tons of equivalent $\mathrm{CO}_{2}$ per tera-joule. Note that $C E F=69.2 \mathrm{~g} / \mathrm{MJ}$ in case of diesel. Therefore, $Z$ is given by: $Z=$ $742826.141 \cdot 10^{3} \quad\left(t \mathrm{CO}_{2} e\right)$.

Furthermore, let us express the emissions of pollutant $h$, expressed in tons of pollutant, as:

$$
P_{h}=E \cdot P E F_{h}
$$

where $E$ is given by (2) and $P E F_{h}$ is the specific emission factor for pollutant gas $h$, expressed in pollutant per tera-joule. In the present work we consider four different types of pollutants, i.e. $h=1$, ..., 4, namely:

- $\quad$ 1: carbon monoxide (CO), such that $P_{C O}=6640.689(t)$;

- 2: nitrogen oxide (NOX), such that $P_{N O_{x}}=1610.172(t)$;

- 3: thin powders (PM), such that $P_{P M}=6640.689(t)$;

- 4: hydrocarbons (HC), such that $P_{H C}=107.345(t)$.

Moreover, let us define 


$$
E^{\prime}=\frac{E}{t} ; Z^{\prime}=\frac{Z}{t} ; P^{\prime}{ }_{h}=\frac{P_{h}}{t}
$$

where $E^{\prime}$ is the energy intensity, expressed in $M J / t-k m, Z^{\prime}$ is the $\mathrm{CO}_{2}$ emission intensity, expressed in $g \mathrm{CO}_{2} \mathrm{e} / \mathrm{t}-\mathrm{km}, \mathrm{P}_{h}{ }^{\prime}$ is the emission intensity of pollutant $h$, expressed in $\mathrm{g} / \mathrm{t}-\mathrm{km}$ and $t$ is the transport efficiency, expressed in $M t / k m$. Note that $E, Z$ and $P_{h}$ are determined by equation (2), (3) and (4), respectively.

From [29] it has been possible to derive the Italian transport efficiency, that is assumed to be $t=$ $117813 \mathrm{Mt} / \mathrm{km}$. Therefore, the values here considered for the quantities given in equation (5) are the following:

$$
\begin{array}{ll}
- & E^{\prime}=91.115 \mathrm{MJ} ; \\
- & Z^{\prime}=6.305 \mathrm{MJ} ; \\
- & P^{\prime}{ }_{\mathrm{CO}}=0.056 \mathrm{MJ} ; \\
- & P^{\prime}{ }_{N O_{x}}=0.014 \mathrm{MJ} ; \\
- & P_{P M}^{\prime}{ }_{P M}=0.056 \mathrm{MJ} ; \\
- & P^{\prime}{ }_{H C}=0.001 \mathrm{MJ} .
\end{array}
$$

Analogously to what has been done for the computation of the energy and pollutant emission intensity given in equations (2) - (4), it has been possible to estimate the intensity of road accidents. It is worth noting that accidents are classified into $f a$, $\sin$ e $m a$, referring, respectively, to fatal accidents, accidents with serious injuries and minor accidents. The road accidents intensity measures the number of accidents of each of the three above types occurred in one year. According to data provided by the Italian Institute of Statistics (Istat [30]), these values related to the Italian road traffic are the following [30]: $f a=3381 ; \sin =15000 ; m a=236147$.

Finally, it is possible to derive the external costs assigning monetary values to both the emission and accident intensities according to the following equation:

$$
E C=10^{-4} \cdot\left(c_{\mathrm{CO}_{2}} \cdot Z^{\prime}+\sum_{h=1}^{4} c_{p_{h}} \cdot P^{\prime}{ }_{h}\right)+10^{2} \cdot\left(c_{f a} \cdot f a+c_{\sin } \cdot \sin +c_{m a} \cdot m a\right) / \mathrm{t}
$$

Equation (6) determines the annual external costs $E C$ due to traffic intensity of the road transport modality, expressed in Euro cents per ton $/ \mathrm{km}$. More precisely, $\mathrm{C}_{\mathrm{CO}_{2}}$ is the unit cost of a ton of $\mathrm{CO}_{2}$ $\left(25 € / t \mathrm{CO}_{2} e\right), c_{p_{h}}$ is the cost of a ton of pollutant $h\left(c_{C O}=0 € / t ; c_{H C}=1242 € / t ; c_{C O_{x}}=10824 € / t ; c_{P M}\right.$ $=197361 € / t)$. Furthermore, according to [23], the annual costs components associated with the accidents are the following: $c_{f a}=1.92 \mathrm{M€}$ is the cost associated with a fatal accident, $c_{m a}=0.02 \mathrm{M€}$ is the cost associated with a minor accident and $c_{\sin }=0.25 \mathrm{M€}$ is the cost associated with an accident with serious injuries. Finally, $Z$ ' and $P^{\prime}{ }_{h}$ are derived from equation (5), while the coefficients $10^{-4}$ and $10^{2}$ are correcting factors of the unit of measures.

Note that, even if it has been possible to derive a unique expression for the external costs, the required cost components involved in such expression are not so easy to get from the literature and could contain approximations. However, equation (6) represents a first attempt to introduce the overall annual cost of externalities due to road transport in Italy. Equation (6), which will be used in the objective function of the optimization model proposed in the next section, has been derived by making reference to data provided by [18] and to recent studies published by Eurostat [29], Istat [30] and Italian Petroleum Union [32]. 


\subsection{The multi-origin multi-destination min cost flow problem with externalities.}

Let us now describe the optimization problem, and the related model, consisting of a multi origin - multi destination min cost flow problem in the presence of external costs associated with the capacitated arcs. From the previous subsection, we know that the external costs are expressed in Euro cents per ton per kilometer. Then, such cost can be easily added to the travelling cost $c_{i j}$ associated with each $\operatorname{arc}(i, j) \in A$ as multiplicative factors of the length $l_{i j}$ of the arcs. If it is the case, we have the new objective function to minimize given by:

$$
C_{E C}=f(\underline{x})=(c+E C) \cdot \sum_{(i, j) \in A} l_{i j} \cdot x_{i j}
$$

where EC is defined in equation (6).

However, the external cost components of the objective function vary based on the amount of flow through the arcs of the network and (7) can be rewritten by opportunely modifying the cost $(c+E C)$. More precisely, we introduce coefficients $c_{1}, c_{2}$ and $c_{3}$ in order to be able to model the different levels of traffic intensity, namely moderate, normal and intense, commonly used in the analysis of the externalities in road transportation network [18]; thus, we represent the fact that increasing the flow also increases both pollution and the risk of road accidents.

In practice, (8) determines the threshold values for which the coefficients of the external costs vary following the estimates made in [18] based on the traffic intensity. Remember that, we refer to $c_{\mathrm{k}}=k E C+c, k=1,2,3$ in case of moderate, normal and intense road traffic, respectively.

Thus, here we propose the following cost function, depending on the value of $\underline{x}$ :

$$
C_{E C}=f(\underline{x})=\left\{\begin{array}{c}
c_{1} \cdot \sum_{(i, j) \in A} l_{i j} \cdot x_{i j} \text { if } x_{i j} \leq 0.5 q_{i j} \\
c_{2} \cdot \sum_{(i, j) \in A} l_{i j} \cdot x_{i j} \text { if } 0.5 q_{i j}<x_{i j} \leq 0.75 q_{i j} \\
c_{3} \cdot \sum_{(i, j) \in A} l_{i j} \cdot x_{i j} \text { if } 0.75 q_{i j}<x_{i j} \leq q_{i j}
\end{array}\right.
$$

Figure 1 reports an example of the resulting new step-wise function (8) for an arc of 100 kilometers whit a capacity of 2000.

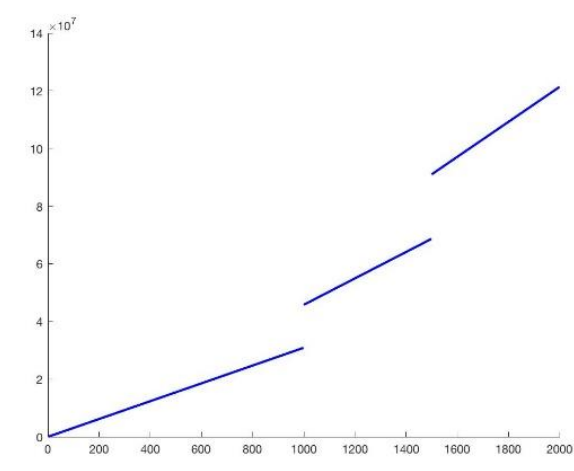

Figure 1: $C_{e c}$ behavior

Therefore, in order to deal with the discontinuity of (8), we solve a new multi origin - multi destination min cost flow problem with additional binary variables $y_{i j}^{1}, y_{i j}^{2}, y_{i j}^{3}$ defined as follows: 


$$
\begin{array}{ll}
y_{i j}^{1}=1 \text { iff } & x_{i j} \leq 0.5 q_{i j}, \forall(i, j) \in A ; \\
y_{i j}^{2}=1 \text { iff } & 0.5 q_{i j}<x_{i j} \leq 0.75 q_{i j}, \forall(i, j) \in A ; \\
y_{i j}^{3}=1 \text { iff } & 0.75 q_{i j}<x_{i j} \leq q_{i j}, \forall(i, j) \in A .
\end{array}
$$

Thus, the function (8) can be re-written as equation (10):

$$
C_{E C}=\sum_{k=1}^{3} c_{k} \sum_{(i, j) \in A}\left(l_{i j} \cdot x_{i j}\right) y_{i j}^{k}
$$

Therefore, the present multi origin - multi destination min cost flow problem with externalities can be formally formulated as follows:

$$
\text { Min } \quad C_{E C}
$$

Subject to

$$
\begin{array}{lc}
\sum_{(i, j) \in A} x_{i j}-\sum_{(l, i)} x_{l i}=b_{i} & \forall i \in N \\
x_{i j} \leq 0.5 q_{i j} y_{i j}^{1}+0.75 q_{i j} y_{i j}^{2}+q_{i j} y_{i j}^{3} & \forall(i, j) \in A \\
\sum_{k=1}^{3} y_{i j}^{k} \leq 1 & \forall(i, j) \in A \\
x_{i j} \geq 0 & \forall(i, j) \in A \\
y_{i j}^{k} \in\{0,1\} & k=1,2,3, \forall(i, j) \in A
\end{array}
$$

where $b_{i}$ in classical flow constraints (12) is given by:

$$
b_{i}=\left\{\begin{array}{c}
g_{i} \forall i \in P \\
-g_{i} \forall i \in D \\
0 \forall i \in T
\end{array}\right.
$$

(13) and (14) are used to define binary variables in accordance with (9); in particular, thanks to (14) at most one binary variables for each arc can be selected. In (15) the decision variables are defined. Note that the objective function (11) can be easily linearized by defining new non-negative variables $z_{i j}^{k}=x_{i j} y_{i j}^{k}, \quad k=1,2,3, \forall(i, j) \in A$ with additional constraints for their definition and linking them to variables $x_{i j}$ and $y_{i j}^{k}$.

\section{Computational experimentation.}

The purpose of the computational experimentation that has been performed and here summarized is twofold. First, we solved the min cost flow problem presented in Section 2.3 using ad-hoc generated random instances, representing road logistic networks, having different size and capacity values; the main aim of this experimentation is to evaluate the impact of the external cost components on the choice of the optimal origin-destination $(o-d)$ routes and the related overall shipping costs. In particular, a comparison between the solutions obtained with and without the externalities is presented and analyzed. Secondly, we wanted to evaluate the road logistic network of the Liguria County, in Italy, connecting its main three ports to the most required inland destinations, to see whether, and how, the cost and the route of the containerized import flow change by varying the level of flow travelling along the arcs due to the external cost components. These two analyses and the related results are reported in the following subsections. 
All the random instances have been generated by using MATLAB, while computational tests have been performed on MPL [31] on a PC Intel(R) Core(TM) i7-6650U CPU, 2.20GHz, $2.21 \mathrm{GHz}, 16$ GB of RAM.

\subsection{Test bed with randomly generated instances.}

Since there are no available benchmark cases in the literature suitable for being a valid test bed for the proposed model (11)-(15), we had the need of generating new sets of instances. In particular, we used MATLAB for implementing a function, denoted $\operatorname{graph}(n, r)$, generating road logistic networks with different characteristics, connecting hypothetical ports to the hinterland. Values $n$ and $r$ are the input parameters of this function, representing, respectively, the number of nodes and the density of the digraph to generate, such that $m \leq r n$ is the actual number of arcs. More precisely, $r$ is a constant, whose value in our experimentation has been fixed to 2, 3 and 4. Then, we set $n$ from 10 to 50. Note that the number $m$ of generated arcs of $G$ is generally lower than its upper bound $r n$ since during the generation of the outgoing arcs from a node both multiple arcs and those that generate loops have not been considered.

Once $n$ is defined, function $\operatorname{origin}-\operatorname{dest}(G, n)$ generates a vector of length $\frac{n}{4}$ and a vector of length $\frac{n}{3}$ containing the origin and destination nodes belonging to $P$ and $D$, respectively, whose number is rounded to the closest integer value of the resulting ratio. These values for the size of $P$ and $D$ have been fixed in such a way to obtain networks with the same density used in the real transport networks we are involved with. All other remaining nodes are the transition nodes belonging to set $T$.

Just to give an idea of the number of arcs effectively generated by function $\operatorname{graph}(n, r)$, Table 1 , describing the characteristics of the generated instances, reports the number of $\operatorname{arcs}$ (column $m$ ) of the randomly generated graphs in each considered instance types (column $i d$ ), given the number of node $(n)$ and the required density $(r)$. Note that we generated five different instances or each $i d$ value.

An example of two randomly generated graphs, one related to an instance of the type id 4 ( $n=20$ and $r=2)$ and the other related to an instance of the type id $5(n=20$ and $r=3)$ is reported in Figure 2.

Table 1. Example of number of arcs effectively generated given the number of nodes $n$

\begin{tabular}{|c|c|c|c|c|}
\hline $\boldsymbol{i d}$ & $\boldsymbol{n}$ & $\boldsymbol{r}$ & $\boldsymbol{r n}$ & $\boldsymbol{m}$ \\
\hline 1 & 10 & 2 & 20 & 19 \\
\hline 2 & 10 & 3 & 30 & 26 \\
\hline 3 & 10 & 4 & 40 & 33 \\
\hline 4 & 20 & 2 & 40 & 38 \\
\hline 5 & 20 & 3 & 60 & 56 \\
\hline 6 & 20 & 4 & 80 & 73 \\
\hline 7 & 30 & 2 & 60 & 57 \\
\hline 8 & 30 & 3 & 90 & 85 \\
\hline 9 & 30 & 4 & 120 & 112 \\
\hline 10 & 50 & 2 & 100 & 98 \\
\hline 11 & 50 & 3 & 150 & 147 \\
\hline 12 & 50 & 4 & 200 & 193 \\
\hline
\end{tabular}



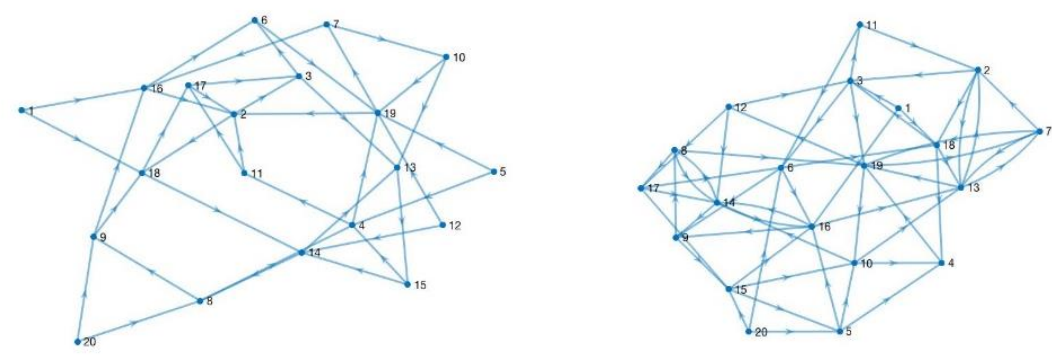

Figure 2: Example of two randomly generated digraphs with $n=20, r=2$ and $n=20, r=3$.

The output of function $\operatorname{graph}(n, r)$ is a digraph $G$ with its properties (generated arcs and their capacities) and its associated distance matrix $L$. Note that $L$ is a sparse matrix whose element $l_{i j}$ represents the length of the already generated $\operatorname{arc}(i, j), \forall(i, j) \in A$. In order to reproduce realistic road logistic networks, we fixed the randomly generated length of each arc between 0 and 100 kilometers.

From $l_{i j}$ we derived the cost of $\operatorname{arc}(i, j), \forall(i, j) \in A$, as in (1), where the constant cost value $c$, depending on the type of vehicle used, is here fixed to $c=1,57 €$ per vehicle per kilometer. Furthermore, from $l_{i j}$ we computed the capacity $q_{i j}$ of arc $(i, j)$ as the maximum number of trucks which can travel along arc $(i, j)$ considering the required minimum safety distance. More precisely, value $q_{i j}$ has been computed as the ratio between $l_{i j}$ and 50, that is the sum of the standard length of a truck transporting containers $(10 \mathrm{~m})$ and the safety distance between two vehicles $(40 \mathrm{~m})$, considering an average travelling speed of $90 \mathrm{~km} / \mathrm{h}$ (interested readers are invited to see [28] for technical insight).

Finally, we generated the amount of units of containerized flow $b$ along the network. Parameter $b$ has been chosen as a percentage of the total flow capacity $Q=\sum_{(i, j) \in A} q_{i j}$ of the network, such that $0.2 Q \leq b \leq 0.5 Q$. The available flow at the origin nodes, which is the same to deliver at the destination nodes, is then randomly assigned to $g_{p}, \forall p \in P$ between 1 and $b / \mid P$.

We implemented model (11)-(15) on MPL and solved it by using GUROBI 7.5.1 as solver.

In order to compare the optimal system costs $\left(\boldsymbol{C}_{\boldsymbol{E} C}\right)$ obtained by model (11)-(15) with the costs of a system focused only to the transportation costs $(\mathbf{C})$ given by (1), we solved the minimum cost flow model with arc capacity. In this last case we minimize (1) subject to the flow conservation constraints (12), capacity constraints (easier than (13), i.e. $x_{i j} \leq q_{i j} \forall(i, j) \in A$ ) and the non-negative conditions for $x_{i j}$ (i.e. $x_{i j} \geq 0, \forall(i, j) \in A$ ).

Table 2 reports a synthesis of our computational experimentation with the randomly generated instances. Column headings are as follows: $(\boldsymbol{i d})$ is the instance type, $(\boldsymbol{C})$ and $\left(\boldsymbol{C}_{\boldsymbol{E} C}\right)$ are the average optimal values of the function (1) and (10), respectively. Analogously, values in columns $(\boldsymbol{L})$ and $\left(\boldsymbol{L}_{E C}\right)$ are the total travelled distance by the trucks when excluding or considering the external costs. Columns $(\Delta \mathbf{C} \%)$ and $(\Delta \mathbf{L} \%)$ give the deviation of the corresponding cost and distance, respectively, between the optimal solution with and without the external costs. Finally, column (Post) reports the value of function (10) evaluated in the optimal flow solution obtained when the minimum cost flow model with arc capacity is solved using function (1). More precisely, (Post) represents the external cost supported by the network when they are not included in the decision process, and $(\Delta \mathbf{P o s t} \%)$ is the corresponding deviation. 
Table 2. Results of the computational experiences with random instances

\begin{tabular}{|c|c|c|c|c|l|l|c|c|}
\hline $\boldsymbol{i d}$ & $\boldsymbol{C}$ & \multicolumn{1}{c|}{$\boldsymbol{C}_{\boldsymbol{E C}}$} & $\boldsymbol{L}$ & \multicolumn{1}{|c|}{$\boldsymbol{L}_{\boldsymbol{E C}}$} & $\boldsymbol{\Delta C}(\boldsymbol{\%})$ & $\boldsymbol{\Delta L}(\boldsymbol{\%})$ & Post & $\Delta$ Post (\%) \\
\hline 1 & 27623 & 81264 & 17594 & 17666 & $194.2 \%$ & $0.4 \%$ & 87477 & $7.6 \%$ \\
\hline 2 & 31219 & 85697 & 19885 & 20718 & $178.1 \%$ & $7.4 \%$ & 101721 & $22.2 \%$ \\
\hline 3 & 58928 & 156487 & 37534 & 38605 & $165.6 \%$ & $2.9 \%$ & 167298 & $6.9 \%$ \\
\hline 4 & 60823 & 140210 & 38741 & 40145 & $130.5 \%$ & $3.6 \%$ & 175240 & $25.0 \%$ \\
\hline 5 & 69248 & 153052 & 44107 & 45667 & $121.0 \%$ & $3.5 \%$ & 174531 & $14.0 \%$ \\
\hline 6 & 86105 & 177478 & 54844 & 56614 & $106.1 \%$ & $3.2 \%$ & 285945 & $61.1 \%$ \\
\hline 7 & 91182 & 181663 & 58078 & 58078 & $99.2 \%$ & $0.0 \%$ & 181663 & $0.0 \%$ \\
\hline 8 & 58304 & 121396 & 37136 & 37159 & $108.2 \%$ & $0.1 \%$ & 121741 & $0.3 \%$ \\
\hline 9 & 118719 & 241060 & 75617 & 77090 & $103.1 \%$ & $1.9 \%$ & 293538 & $21.8 \%$ \\
\hline 10 & 388953 & 792522 & 247741 & 248743 & $103.3 \%$ & $0.3 \%$ & 814039 & $2.8 \%$ \\
\hline 11 & 270775 & 534047 & 172468 & 174290 & $97.2 \%$ & $1.1 \%$ & 588450 & $10.2 \%$ \\
\hline 12 & 183170 & 403230 & 116669 & 121713 & $120.1 \%$ & $4.3 \%$ & 481174 & $19.3 \%$ \\
\hline $\boldsymbol{A v g}$ & $\mathbf{1 2 0 4 2 1}$ & $\mathbf{2 5 5 6 7 5}$ & $\mathbf{7 6 7 0 1}$ & $\mathbf{7 8 0 4 1}$ & $\mathbf{1 2 7 . 2 \%}$ & $\mathbf{2 . 4 \%}$ & $\mathbf{2 8 9 4 0 1}$ & $\mathbf{1 5 . 9 \%}$ \\
\hline
\end{tabular}

Looking at Table 2 it is possible to note that, on average, the kilometers travelled when the external costs are included in the decision process $\left(\boldsymbol{L}_{\boldsymbol{E C}}\right)$ are higher of about $2.4 \%$ than the $\boldsymbol{L}$ ones. In fact, according to model (11)-(15) the best paths are those corresponding to a trade-off between distance and flow intensity, that is longer routes allow to reduce the traffic intensity and hence the cost components of equation (8). For a better understanding, look at the row of instance id 6 , column $C_{E C}$, corresponding to the best results of model (11)-(15); in order to reduce of about $60 \%$ the total transport costs (Post), it modifies the shortest paths with an increment of the travelled kilometers of about 3.2\% $(\Delta L)$. On the opposite, the worst case in terms of kilometers travelled is represented by instance id 2, in which the length of the paths is increased of about $7.4 \%$ in order to reduce the overall costs of about $22 \%$.

Figure 3 depicts and compares the costs of the system $\left(\boldsymbol{C}, \boldsymbol{C}_{\boldsymbol{E} C}\right.$ and Post $)$. Post for all instances, except for instance id 7, is larger than $\boldsymbol{C}_{\boldsymbol{E} C \boldsymbol{C}}$. Furthermore, note that instances with $\Delta P$ Post lower than $15 \%$ (id $1,3,5,7,8,10,11$ ) are those characterized by a low demand with respect to the network capacity.

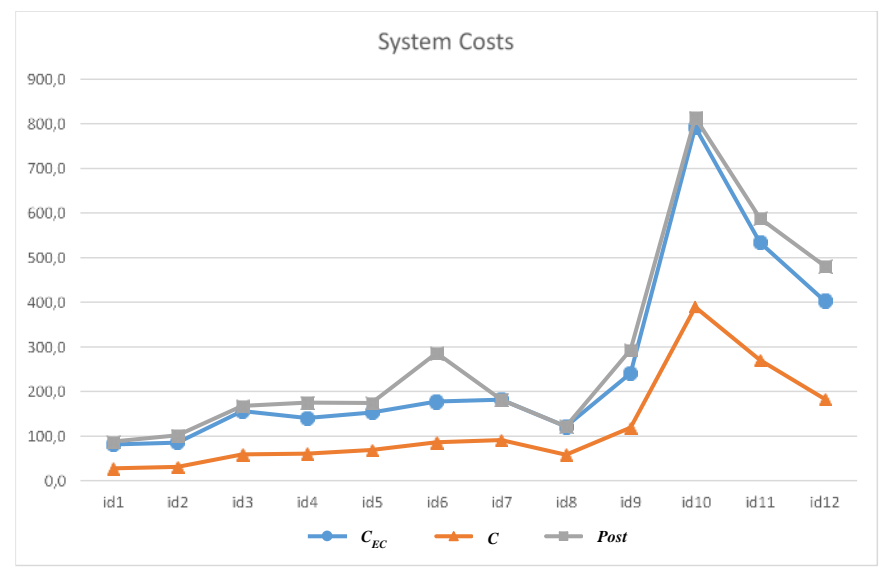

Figure 3: a comparison of the system costs (costs/1000) 


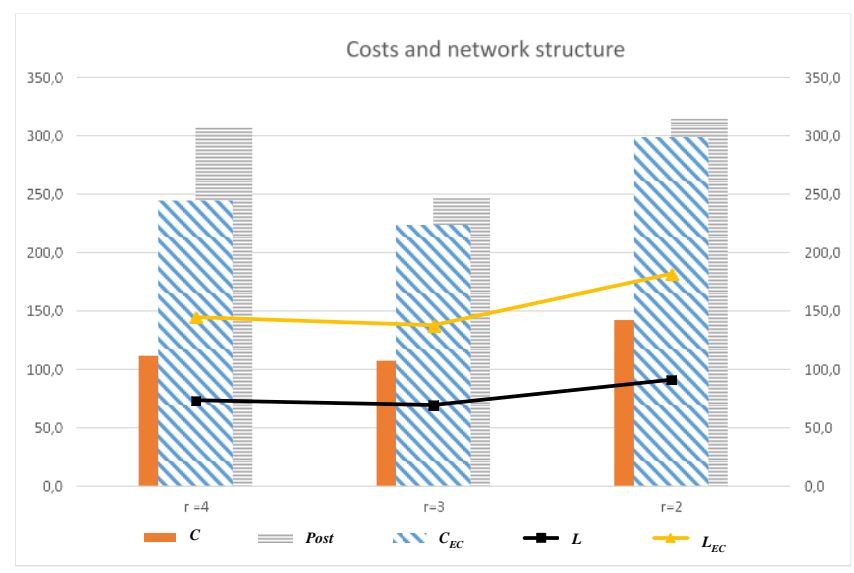

Figure 4: the system costs in different networks

Figure 4 shows how the costs and the distances are influenced by the density $r$ of the network structure. In a network characterized by few connections $(r=2)$ the external costs are more impacting, also in case they are included in the decision process (i.e. the difference between $\boldsymbol{C}_{\boldsymbol{E} C}$ and Post is low and the transport cost $\boldsymbol{C}$ is high). The greater difference between $\boldsymbol{C}_{\boldsymbol{E} C}$ and Post results in a more connected network (i.e. $r=4$ ). Summarizing it emerges that a transport policy finalized to the inclusion of the external costs in the decision process can be usefully adopted in well-connected networks.

Figures 5, 6, 7 and 8 give an idea about how the $o$ - $d$ paths change (and consequently the flows along arcs) when minimizing the sum of transport costs (1) and the sum of transportation and external costs (10).

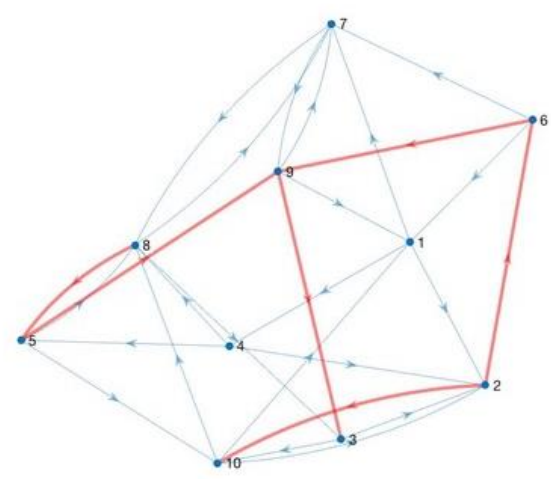

(a)

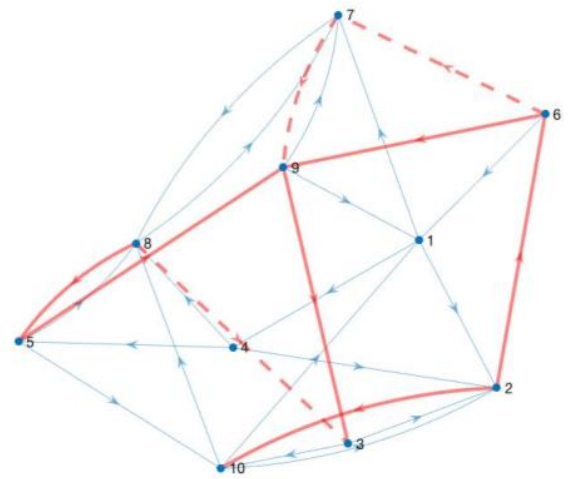

(b)

Figure 5: optimal solutions instance Id2 (a) cost function (1); (b) with external costs, cost function (10) 


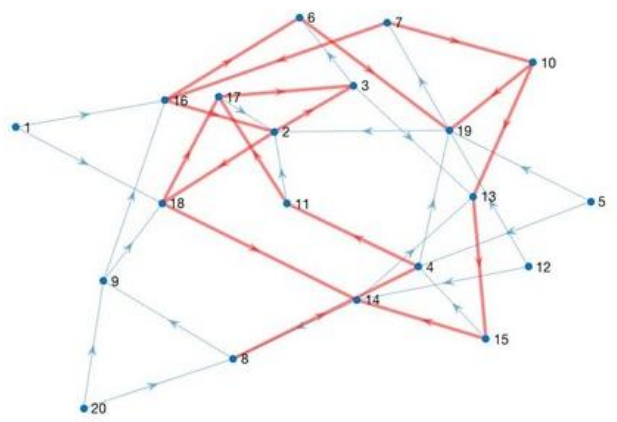

(a)

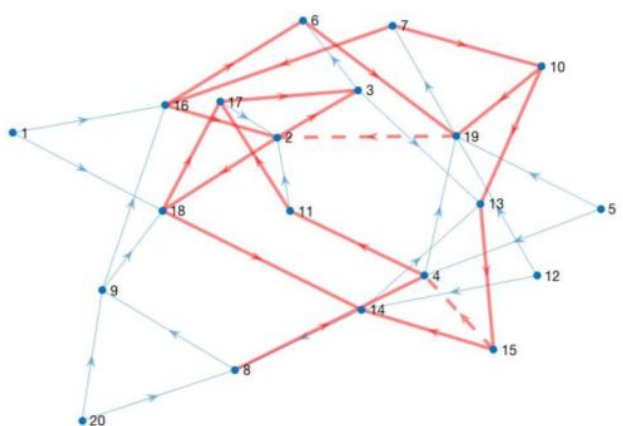

(b)

Figure 6: optimal solutions instance Id4 (a) cost function (1), (b) with external costs, cost function (10)

Dashed arcs in Figures 5 (b), 6 (b) represent new connections chosen for minimizing transportation and external costs of equation (10).

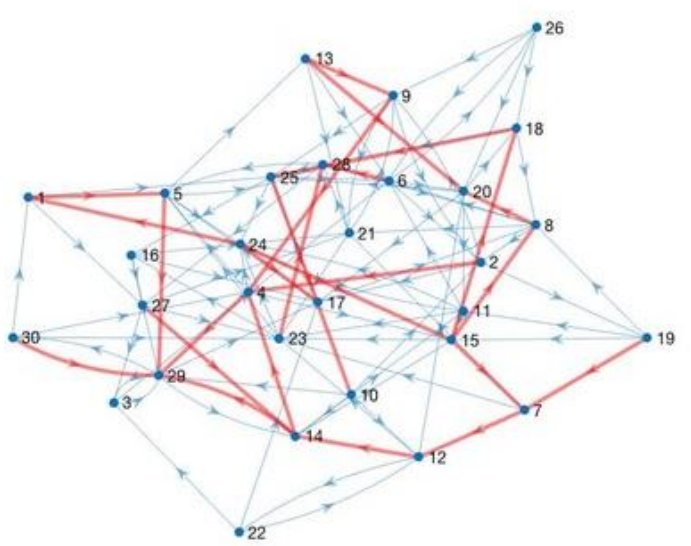

(a)

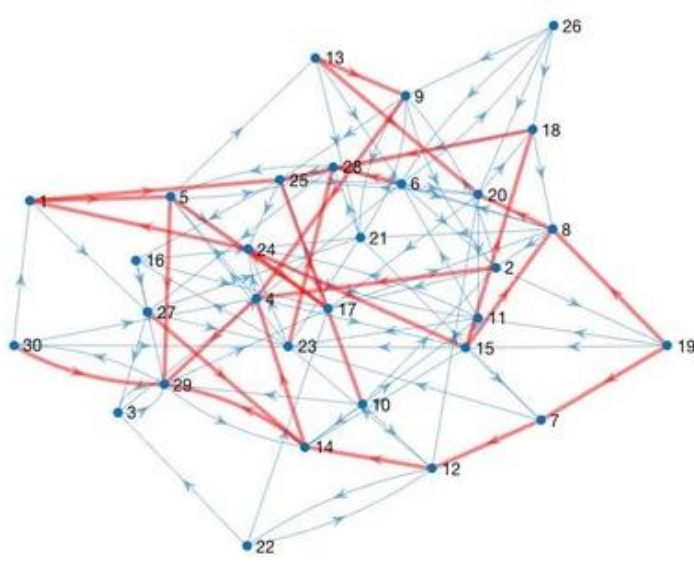

(b)

Figure 7: optimal solutions instance Id9 (a) cost function (1), (b) with external costs, cost function (10)

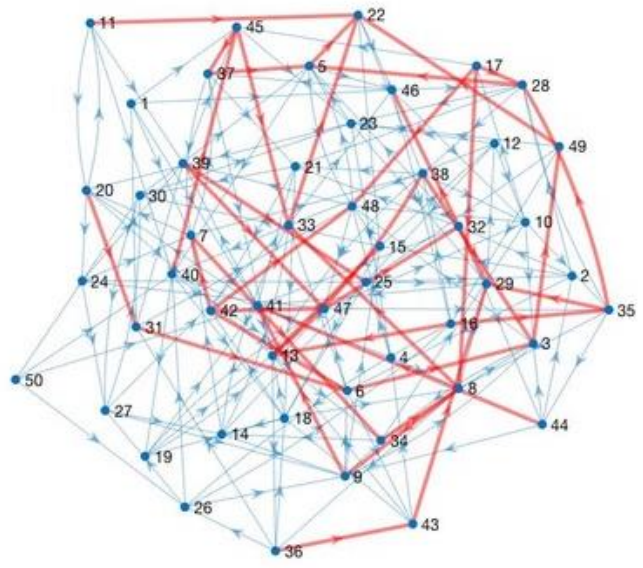

(a)

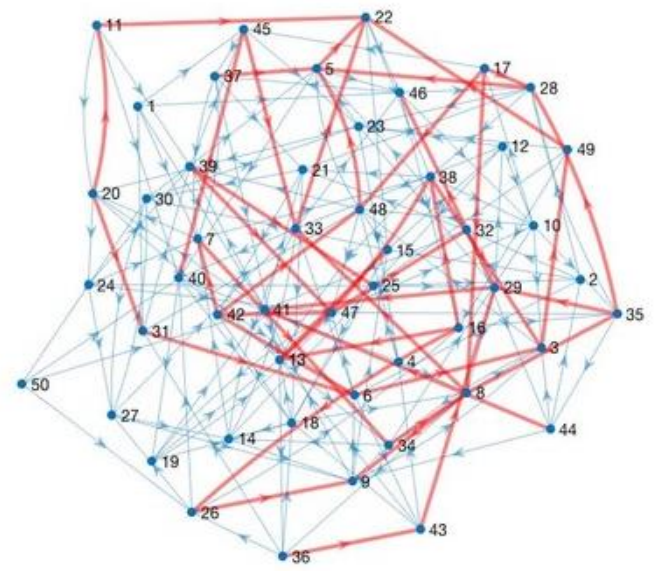

(b)

Figure 8: optimal solutions instance I12 (a) cost function (1), (b) with external costs, cost function (10)

\subsection{Case study: the Ligurian ports logistic network.}

The second part of the proposed computational experimentation is devoted to the analysis of the freight distribution network from three of the major Italian ports, that is Genoa, La Spezia and Savona, 
located in Liguria County. Note that, from 2014 the Ligurian ports accounted for about $35 \%$ of the Italian container traffic, being the greatest gateway port region in Italy [2]. In particular, our aim is to evaluate the impact of the externalities, due to pollution and accidents, in the road network of the Italian north-western geographical area used by trucks for shipping the containerized flow departing from maritime terminals at the three above mentioned ports toward the hinterland for their forwarding to the final destinations or the main logistics corridors.

Set $P$ hence consists of port of Genova, port of Savona and port of La Spezia, while set $D$ consists of the cities Alessandria, Aosta, Brescia, Como, Cuneo, Genova, La Spezia, Milano, Parma, Savona, Torino, Varese and Verbania. Set $T$ contains the intersection points in the highway and other cities, such that $|\mathrm{T}|=22$. The road network connecting sets $P$ and $D$ is reported in Figure 9. The network model generated from the input data concerning the road map and traffic information is reported in Figure 10.

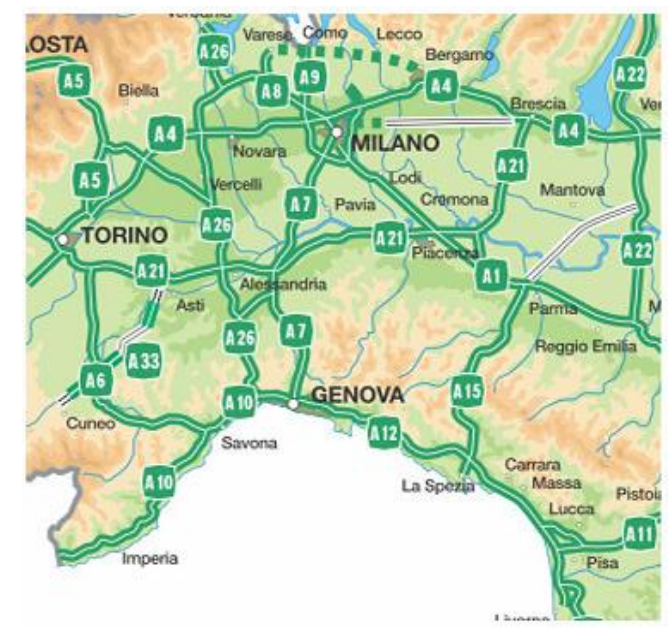

Figure 9: The Ligurian highway road map.

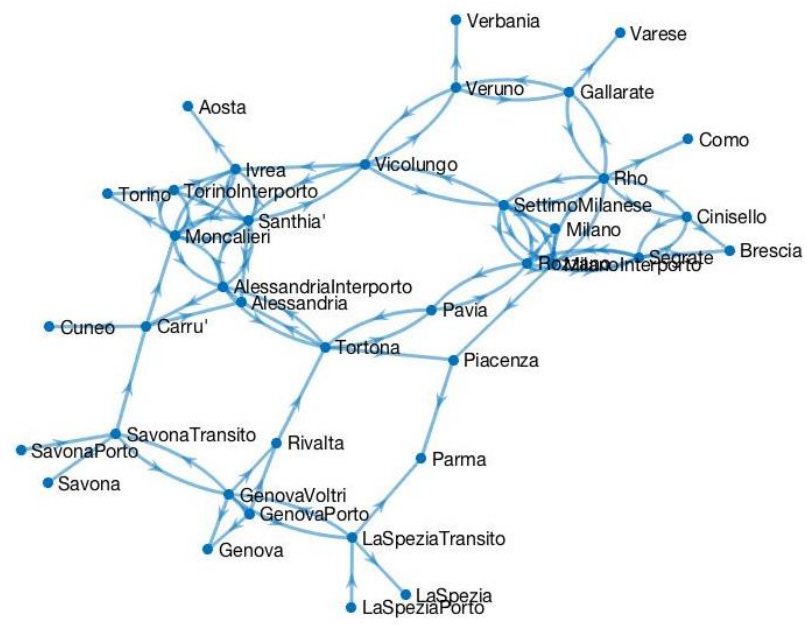

Figure 10: The network model of the Ligurian logistics network. 
We solved the problem for the real network considering different levels of $o$ - $d$ demands D1, D2, D3 and D4. In particular, the demand ranges between 450000 (D1) and 9000000 (D4) containers that are about $1 \%$ and $45 \%$ of the out flow capacity of the node belonging to set $P$.

The obtained results are presented in Table 3; looking for example at the last row of Table 3 is evident that by introducing the external cost in the decision process it is possible to reduce the costs of the system by more than $37 \%$, but as expected the length of the paths increase of about $12 \%$.

Table 3. Results of the computational experiences with real case instances.

\begin{tabular}{|c|c|c|c|c|c|c|c|c|}
\hline id & $\boldsymbol{C}_{\boldsymbol{E C}}$ & $\boldsymbol{C}$ & $\boldsymbol{L}_{E C}$ & $\boldsymbol{L}$ & $\Delta \boldsymbol{C}(\boldsymbol{\%})$ & $\Delta \boldsymbol{L}(\boldsymbol{\%})$ & $\boldsymbol{P o s t}$ & $\Delta$ Post $(\boldsymbol{\%})$ \\
\hline$D 1$ & 272863 & 140044 & 89200178 & 89200178 & $94.84 \%$ & $0.00 \%$ & 273733 & $0.32 \%$ \\
\hline$D 2$ & 2728639 & 1400446 & 892003571 & 892003571 & $94.84 \%$ & $0.00 \%$ & 2737337 & $0.32 \%$ \\
\hline$D 3$ & 6114136 & 2800891 & 1998736808 & 1784006795 & $118.29 \%$ & $11.48 \%$ & 8433032 & $37.93 \%$ \\
\hline$D 4$ & 16339213 & 6276033 & 4456424340 & 3997472966 & $160.34 \%$ & $12.04 \%$ & 19797700 & $21.17 \%$ \\
\hline
\end{tabular}

Looking at the graph in Figure 11 it is possible to note that the more saturated is the network, the greater is the difference obtained in the transport decisions when including external costs in the decision process concerning the optimal choice of the route. Note that in Figure 11 as in Table 3 values $\boldsymbol{C}_{\boldsymbol{E} C}, \boldsymbol{C}$ and $\boldsymbol{P o s t}$ are divided by 1000 for scale factors.

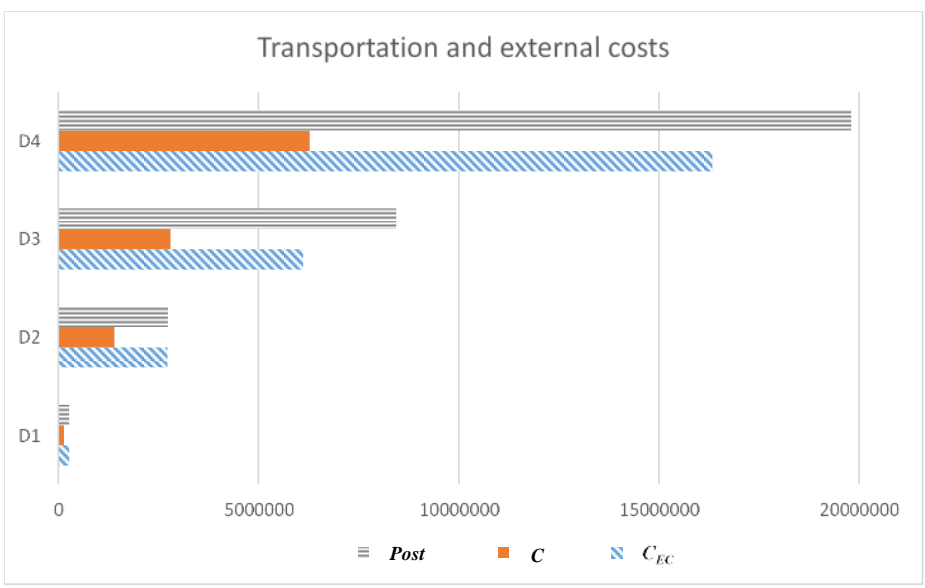

Figure 11: a comparison of the costs in the different demand scenarios (costs/1000)

In fact, in case of low transport demand the inclusion of the external costs in the decision process has a limited effect also on the external costs paid by the collectivity because the arcs have a low level of congestion. The kilometers associated with the solutions obtained by solving model (11)-(15) and by solving the flow problem without external cost are equal in the cases of D1 and D2.

Finally, Figures 12 and 13 give a comparison of the flows distribution in case of instances characterized by high demands (D3 and D4). 


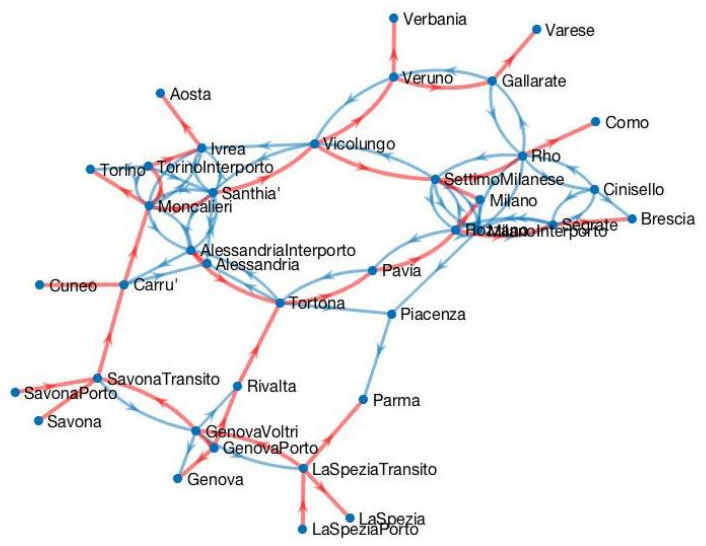

(a)

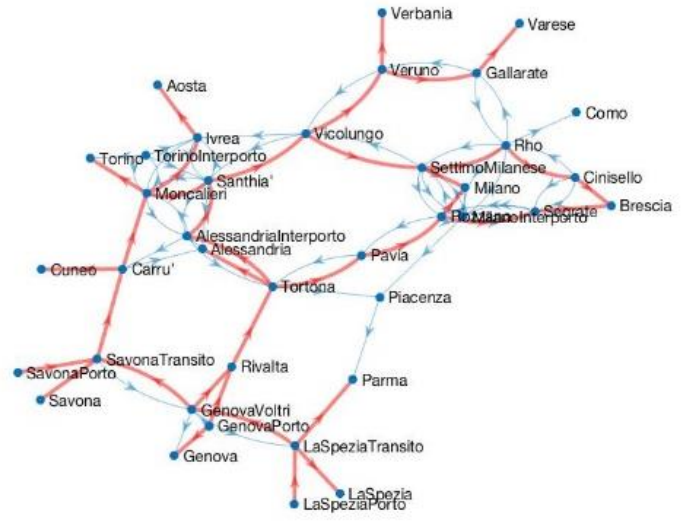

(b)

Figure 12: optimal solutions real instance D3 (a) cost function (1); (b) with external costs, cost function (10).

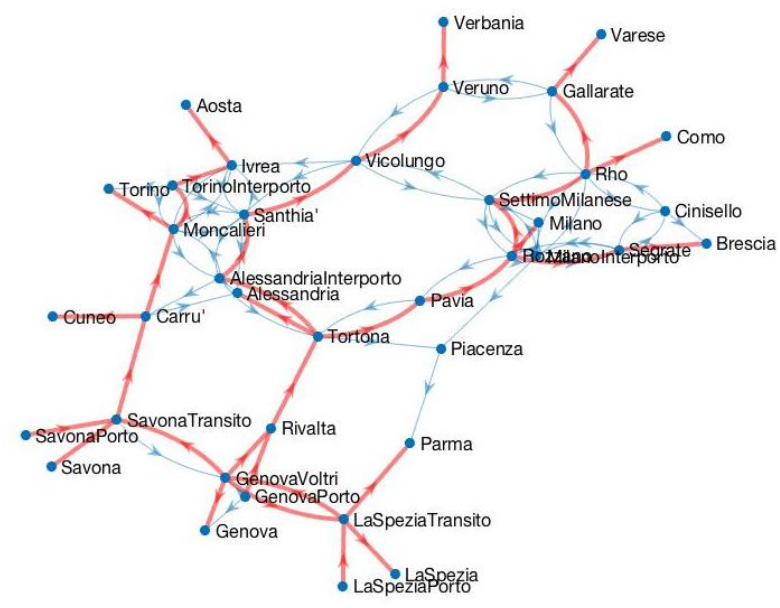

(a)

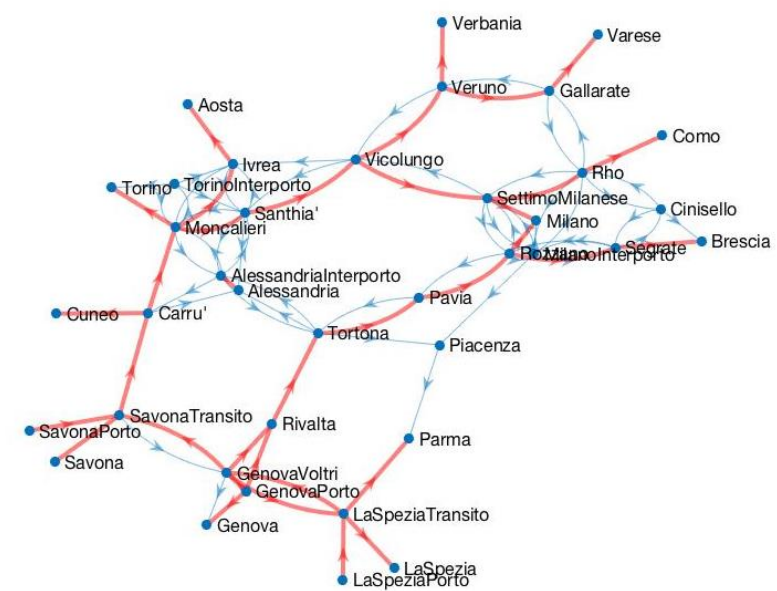

(b)

Figure 13: optimal solutions real instance D4 (a) cost function (1); (b) with external costs, cost function (10).

\section{Conclusions}

In this paper we faced a distribution problem arising in a freight logistics context and we included in the analysis accidental and polluting costs as external negative costs impacting on the collectivity. We used a novel stepwise function for including external costs in the decision process. We evaluated the impact of environmental externalities on the choice of the route in terms of costs and distances depending on the density of the traffic. It resulted that: i) in case of low transport demand the inclusion of the external costs in the decision process has a limited effect also on the external costs paid by the collectivity because the arcs have a low level of congestion; ii) a transport policy finalized to the inclusion of the external costs in the decision process can be usefully adopted in well-connected networks. 
Acknowledgements. This work has been partially supported by PRIN 2015 research project funded by the Italian Ministry of Education, University and Research: SPORT: Smart PORt Terminals Gate Operations and inland forwarding.

\section{References}

[1] D. Ambrosino, A. Sciomachen: Investments evaluation in seaports for increasing the rail split modality in multimodal freight networks, SOLI (2017), IEEEXplore, pp.181-186.

[2] D. Ambrosino, C. Ferrari, A. Sciomachen, A. Tei: Intermodal and external costs: Re-thinking the current network organization, Research in Transportation Business \& Management, 19, 106-117 (2016).

[3] P. Arnold, D. Peeters, I. Thomas: Modelling a rail/road intermodal transportation system, Transportation Research Part E, 40,3, 255-270 (2004).

[4] Brandenburg M., Govindan K., Sarkis J., Seuring S.: Quantitative models for sustainable supply chain management: Developments and directions. EJOR, 233, 299-312 (2014).

[5] Carrabs F, Cerulli R, Sciomachen A.: Environmental sustainable fleet planning in B2C ecommerce Urban distribution networks. In: Dameri R, Rosenthal-Sabroux C (eds) Smartcity. How to create public and economic value with high technology in urban space. Springer, Berlin (2014).

[6] Cattaruzza D, Feillet D, Gonzlez-Feliu J.: Vehicle routing problems for city logistics. EURO J Transp Log, 129. doi:10.1007/ s13676-014-0074-0 (2015).

[7] R. Cerulli, P. Dameri, A. Sciomachen: Operations management in distribution networks within a smartcity framework, IMA Journal Management \& Mathematics, 00, 1-17 (2017).

[8] T.G.Crainic, M.Gendreau, J.Potvin: Intelligent freight-transportation systems: Assessment and the contribution of operations research, Transportation Research C 17, 541557 (2009).

[9] T.G. Crainic, N. Ricciardi, G. Storchi: Models for evaluating and planning city logistics systems. Transport. Sci., 43, 432454, (2009).

[10]P.W.De Langed, A.Chouly: Hinterland access regimes in seaports, European Journal of Transport and Infrastructure Research, V. 4 N. 4, 361-80 (2004).

[11]European Environment Agency: Towards a resource-efficient transport system TERM 2009: indicators tracking transport and environment in the European Union, EEA report, n.2, Bruxelles (2010).

[12]F. Iannone: The private and social cost efficiency of port hinterland container distribution through a regional logistics system, Transportation Research Part A, 46, 9, pp. 14241448 (2012).

[13] Govindam K, Sarkis J, Chiappetta Jabbour CJ, Zhu K, Geng Y Eco-efficiency based green supply chain management: current status and opportunities. Eur J Oper Res 2014 (233), 293298 (2014).

[14]Kopfer W, Schnberger J, Kopfer H Reducing greenhouse gas emissions of a heterogeneous vehicle fleet. Flex Serv Manuf J 26(1), 221248 (2014).

[15]M. Janic: Modelling the full costs of an intermodal and road freight transport network, Transportation Research Part D, 12, 1, pp. 3344 (2007).

[16] T. Lakshmanan, P. Nijkamp, P. Rietveld, E.T. Verhoef: Benefits and costs of transport: Classification, methodologies and policies, Papers in Regional Science, 80, 2, pp 139-164 (2001). 
[17]Macharis C., Van Hoeck E., Pekin E., Van Lier T.: A decision analysis framework for intermodal transport: Comparing fuel price increases and the internalisation of external costs, Transportation Research Part A: Policy and Practice 44, 7, 550-561 (2010).

[18]M. Maibach, C. Schreyer, D. Sutter, H.P. van Essen, B.H. Boon, R. Smokers, A. Schroten, C. Doll, B. Pawlowska, M. Bak: Handbook on estimation of external costs in the transport sector, Internalisation Measures and Policies for All External Cost of Transport, IMPACT Project, Version 1.1, European Commission DG TREN, CE Delft, The Netherlands (2008).

[19]T.Notteboom, J.P.Rodrigue Containerization, box logistics and global supply chains: the integration of ports and liner shipping networks, Maritime Economics and Logistic, 10, pp. 152174 (2008).

[20] Mostert M., Caris A., Limbourg A. Road and intermodal transport performance: the impact of operational costs and air pollution external costs, Reserach In Transportation \& Business Management, 23, 75-85 (2017).

[21]P.J. Perez-Martinez, J.M. Vassallo-Magro: Changes in the external costs of freight surface transport in Spain, Research In Transportation Economics, 42, 61-76 (2013).

[22] Petro F, Konecy V. Calculation of emission from transport services and their use for the internalisation of external costs in road transport, Procedia Engineering, 192, 677-682 (2017).

[23] Ricardo-AEA: Update of handbook on external costs of transport (2014).

[24] Santos, G., Behrendt, H., Maconi, L., Shirvani, T., Teytelboymb, A. Part I: externalities and economic policies in road transport. Res. Transp. Econ. 28, 245 (2010).

[25] Santos G. Road fuel taxes in Europe: do they internalize road transport externalities? Transport Policy, 53, 120-134 (2017).

[26] Sarkis J, Zhu Q, Lai KH An organizational theoretic review of green supply chain management literature. Int J Prod Econ 130(1), 115 (2011).

[27] Taniguchi, E., Thompson, R.G. Yamada, T. Emerging techniques for enhancing the practical application of city logistics models . Procedia Soc. Behavior. Sci., 39, 318 (2012).

[28] Automobile Club Italia, www.aci.it.

[29]Eurostat, www.ec.europa.eu/eurostat.

[30] Istat, www.istat.it.

[31] Maximal Software, www.maximal-usa.com

[32] Unione Petrolifera, www.unionepetrolifera.it. 